\title{
On the Number of Topological Types Occurring in a Parameterized Family of Arrangements
}

\author{
Saugata Basu
}

Received: 22 April 2007 / Revised: 22 March 2008 / Accepted: 12 April 2008 /

Published online: 17 May 2008

(C) Springer Science+Business Media, LLC 2008

Abstract Let $\mathcal{S}(\mathbb{R})$ be an o-minimal structure over $\mathbb{R}, T \subset \mathbb{R}^{k_{1}+k_{2}+\ell}$ a closed definable set, and

$$
\pi_{1}: \mathbb{R}^{k_{1}+k_{2}+\ell} \rightarrow \mathbb{R}^{k_{1}+k_{2}}, \quad \pi_{2}: \mathbb{R}^{k_{1}+k_{2}+\ell} \rightarrow \mathbb{R}^{\ell}, \quad \pi_{3}: \mathbb{R}^{k_{1}+k_{2}} \rightarrow \mathbb{R}^{k_{2}}
$$

the projection maps as depicted below:

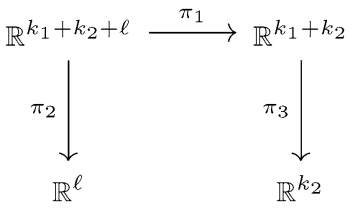

For any collection $\mathcal{A}=\left\{A_{1}, \ldots, A_{n}\right\}$ of subsets of $\mathbb{R}^{k_{1}+k_{2}}$, and $\mathbf{z} \in \mathbb{R}^{k_{2}}$, let $\mathcal{A}_{\mathbf{z}}$ denote the collection of subsets of $\mathbb{R}^{k_{1}}$

$$
\left\{A_{1, \mathbf{z}}, \ldots, A_{n, \mathbf{z}}\right\}
$$

where $A_{i, \mathbf{z}}=A_{i} \cap \pi_{3}^{-1}(\mathbf{z}), 1 \leq i \leq n$. We prove that there exists a constant $C=C(T)>0$ such that for any family $\mathcal{A}=\left\{A_{1}, \ldots, A_{n}\right\}$ of definable sets, where each $A_{i}=\pi_{1}\left(T \cap \pi_{2}^{-1}\left(\mathbf{y}_{i}\right)\right)$, for some $\mathbf{y}_{i} \in \mathbb{R}^{\ell}$, the number of distinct stable homotopy types amongst the arrangements $\mathcal{A}_{\mathbf{z}}, \mathbf{z} \in \mathbb{R}^{k_{2}}$ is bounded by $C \cdot n^{\left(k_{1}+1\right) k_{2}}$ while the number of distinct homotopy types is bounded by $C \cdot n^{\left(k_{1}+3\right) k_{2}}$. This generalizes to the o-minimal setting, bounds of the same type proved in Basu and Vorobjov

The author was supported in part by NSF grant CCF-0634907.

S. Basu $(\bowtie)$

School of Mathematics, Georgia Institute of Technology, Atlanta, GA 30332, USA

e-mail: saugata.basu@math.gatech.edu 
(J. Lond. Math. Soc. (2) 76(3):757-776, 2007) for semi-algebraic and semi-Pfaffian families. One technical tool used in the proof of the above results is a pair of topological comparison theorems reminiscent of Helly's theorem in convexity theory. These theorems might be of independent interest in the quantitative study of arrangements.

Keywords Combinatorial complexity · O-minimal structures · Homotopy types · Arrangements

\section{Introduction}

The study of arrangements is a very important subject in discrete and computational geometry, where one studies arrangements of $n$ subsets of $\mathbb{R}^{k}$ (often referred to as objects of the arrangements) for fixed $k$ and large values of $n$ (see [1] for a survey of the known results from this area). The precise nature of the objects in an arrangement will be discussed in more detail later. Common examples consist of arrangements of hyperplanes, balls or simplices in $\mathbb{R}^{k}$. More generally one considers arrangements of objects of "bounded description complexity". This means that each set in the arrangement is defined by a first-order formula in the language of ordered fields involving at most a constant number of polynomials whose degrees are also bounded by a constant (see [12]).

This paper considers parameterized families of arrangements. The question we are interested in most is the number of "topologically" distinct arrangements that can occur in such a family [a precise definition of the topological type of an arrangement is given later (see Definition 1.6)]. Parameterized arrangements occur quite frequently in practice. For instance, take any arrangement $\mathcal{A}$ in $\mathbb{R}^{k_{1}+k_{2}}$ and let $\pi: \mathbb{R}^{k_{1}+k_{2}} \rightarrow \mathbb{R}^{k_{2}}$ be the projection on the last $k_{2}$ coordinates. Then for each $\mathbf{z} \in \mathbb{R}^{k_{2}}$, the intersection of the arrangement $\mathcal{A}$ with the fiber $\pi^{-1}(\mathbf{z})$, is an arrangement $\mathcal{A}_{\mathbf{z}}$ in $\mathbb{R}^{k_{1}}$ and the family of the arrangements $\left\{\mathcal{A}_{\mathbf{z}}\right\}_{\mathbf{z} \in \mathbb{R}^{k_{2}}}$ is an example of a parameterized family of arrangements. Even though the number of arrangements in the family $\left\{A_{\mathbf{z}}\right\}_{\mathbf{z} \in \mathbb{R}^{k_{2}}}$ is infinite, it follows from Hardt's triviality theorem generalized to o-minimal structures (see Theorem 4.2) that the number of "topological types" occurring among them is finite and can be effectively bounded in terms of the $n, k_{1}, k_{2}$ up to multiplication by a constant that depends only on the particular family from which the objects of the arrangements are drawn. If by topological type we mean homeomorphism type, then the best-known upper bound on the number of types occurring is doubly exponential in $k_{1}, k_{2}$. However, if we consider the weaker notion of homotopy type, then we obtain a singly exponential bound. We conjecture that a singly exponential bound also holds for homeomorphism types as well.

We now make precise the class of arrangements that we consider and also the notion of topological type of an arrangement.

\subsection{Combinatorial Complexity in O-minimal Geometry}

In order to put the study of the combinatorial complexity of arrangements in a more natural mathematical context, as well as to elucidate the proofs of the main results 
in the area, a new framework was introduced in [2] which is a significant generalization of the settings mentioned earlier. We recall here the basic definitions of this framework from [2], referring the reader to the same paper for further details and examples.

We first recall an important model theoretic notion - that of o-minimality-which plays a crucial role in this generalization.

\subsubsection{O-minimal Structures}

O-minimal structures were invented and first studied by Pillay and Steinhorn in the pioneering papers $[13,14]$ motivated by prior work of van den Dries [19]. Later the theory was further developed through contributions of other researchers, most notably van den Dries, Wilkie, Rolin, and Speissegger among others [15, 21-23, 26, 27]. We particularly recommend the book by van den Dries [20] and the notes by Coste [6] for an easy introduction to the topic as well as the proofs of the basic results that we use in this paper.

Definition 1.1 (O-minimal structure) An o-minimal structure over a real closed field $\mathrm{R}$ is a sequence $\mathcal{S}(\mathrm{R})=\left(\mathcal{S}_{n}\right)_{n \in \mathbb{N}}$, where each $\mathcal{S}_{n}$ is a collection of subsets of $\mathrm{R}^{n}$ (called the definable sets in the structure) satisfying the following axioms (following the exposition in [6]).

(1) All algebraic subsets of $\mathrm{R}^{n}$ are in $\mathcal{S}_{n}$.

(2) The class $\mathcal{S}_{n}$ is closed under complementation and finite unions and intersections.

(3) If $A \in \mathcal{S}_{m}$ and $B \in \mathcal{S}_{n}$, then $A \times B \in \mathcal{S}_{m+n}$.

(4) If $\pi: \mathrm{R}^{n+1} \rightarrow \mathrm{R}^{n}$ is the projection map on the first $n$ coordinates and $A \in \mathcal{S}_{n+1}$, then $\pi(A) \in \mathcal{S}_{n}$.

(5) The elements of $\mathcal{S}_{1}$ are finite unions of points and intervals. (Note that these are precisely the subsets of $\mathbb{R}$ which are definable by a first-order formula in the language of the reals with one free variable.)

The class of semi-algebraic sets is one obvious example of such a structure, but in fact there are much richer classes of sets which have been proved to be o-minimal (see $[6,20])$.

\subsubsection{Admissible Sets}

We now recall from [2] the definition of the class of sets that will play the role of sets with bounded description complexity mentioned above.

Definition 1.2 (Admissible sets) Let $\mathcal{S}(\mathbb{R})$ be an o-minimal structure over $\mathbb{R}$ and let $T \subset \mathbb{R}^{k+\ell}$ be a fixed definable set. Let $\pi_{1}: \mathbb{R}^{k+\ell} \rightarrow \mathbb{R}^{k}$ (respectively, $\pi_{2}$ : 
$\mathbb{R}^{k+\ell} \rightarrow \mathbb{R}^{\ell}$ ) be the projections onto the first $k$ (respectively, last $\ell$ ) coordinates.

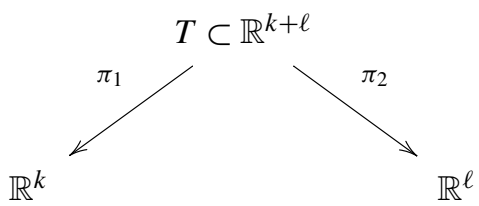

We will call a subset $S$ of $\mathbb{R}^{k}$ to be a $\left(T, \pi_{1}, \pi_{2}\right)$-set if

$$
S=T_{\mathbf{y}}=\pi_{1}\left(\pi_{2}^{-1}(\mathbf{y}) \cap T\right)
$$

for some $\mathbf{y} \in \mathbb{R}^{\ell}$.

If $T$ is some fixed definable set, we call a family of $\left(T, \pi_{1}, \pi_{2}\right)$-sets to be a $\left(T, \pi_{1}, \pi_{2}\right)$-family. We will also refer to a finite $\left(T, \pi_{1}, \pi_{2}\right)$-family as an arrangement of $\left(T, \pi_{1}, \pi_{2}\right)$-sets.

\subsection{Stable Homotopy Equivalence}

For any finite CW-complex $X$ we denote by $\mathbf{S} X$ the suspension of $X$ and for $n \geq 0$,

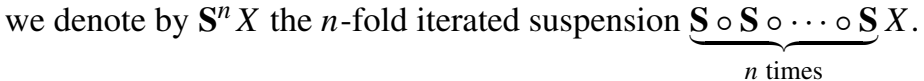

Note that if $i: X \hookrightarrow Y$ is an inclusion map, then there is an obvious induced inclusion map $\mathbf{S}^{n} i: \mathbf{S}^{n} X \hookrightarrow \mathbf{S}^{n} Y$ between the $n$-fold iterated suspensions of $X$ and $Y$.

Recall from [18] that for two finite CW-complexes $X$ and $Y$, an element of

$$
\{X ; Y\}=\underset{i}{\lim }\left[\mathbf{S}^{i} X, \mathbf{S}^{i} Y\right]
$$

is called an $S$-map (or map in the suspension category). An S-map $f \in\{X ; Y\}$ is represented by the homotopy class of a map $f: \mathbf{S}^{N} X \rightarrow \mathbf{S}^{N} Y$ for some $N \geq 0$.

Definition 1.3 (Stable homotopy equivalence) An S-map $f \in\{X ; Y\}$ is an Sequivalence (also called a stable homotopy equivalence) if it admits an inverse $f^{-1} \in\{Y ; X\}$. In this case we say that $X$ and $Y$ are stable homotopy equivalent.

If $f \in\{X ; Y\}$ is an S-map, then $f$ induces a homomorphism

$$
f_{*}: \mathrm{H}_{*}(X, \mathbb{Z}) \rightarrow \mathrm{H}_{*}(Y, \mathbb{Z})
$$

between the homology groups of $X$ and $Y$.

The following theorem characterizes stable homotopy equivalence in terms of homology.

Theorem 1.4 [8, pp. 604] Let $X$ and $Y$ be two finite $C W$-complexes. Then $X$ and $Y$ are stable homotopy equivalent if and only if there exists an $S$-map $f \in\{X ; Y\}$ which induces isomorphisms $f_{*}: \mathrm{H}_{*}(X, \mathbb{Z}) \rightarrow \mathrm{H}_{*}(Y, \mathbb{Z})$. 


\subsection{Diagrams and Co-limits}

The arrangements that we consider are all finitely triangulable. In other words, the union of objects of an arrangement is homeomorphic to a finite simplicial complex, and each individual object in the arrangement will correspond to a subcomplex of this simplicial complex. It will be more convenient to work in the category of finite regular cell complexes, instead of just simplicial complexes.

Let $\mathcal{A}=\left\{A_{1}, \ldots, A_{n}\right\}$, where each $A_{i}$ is a subcomplex of a finite regular cell complex. We will denote by $[n]$ the set $\{1, \ldots, n\}$ and for $I \subset[n]$ we will denote by $\mathcal{A}^{I}$ (respectively, $\mathcal{A}_{I}$ ) the regular cell complexes $\bigcup_{i \in I} A_{i}$ (respectively, $\bigcap_{i \in I} A_{i}$ ). Notice that if $J \subset I \subset[n]$, then

$$
\begin{aligned}
& \mathcal{A}^{J} \subset \mathcal{A}^{I}, \\
& \mathcal{A}_{I} \subset \mathcal{A}_{J} .
\end{aligned}
$$

We will call the collection of sets $\left\{\left|\mathcal{A}_{I}\right|\right\}_{I \subset[n]}$ together with the inclusion maps $i_{I, J}:\left|\mathcal{A}_{I}\right| \hookrightarrow\left|\mathcal{A}_{J}\right|, J \subset I$, the diagram of $\mathcal{A}$. Notice that (even though we do not use this fact), $\left|\mathcal{A}^{[n]}\right|$ is the co-limit of the diagram of $\mathcal{A}$. For $I \subset[n]$ we will denote by $\mathcal{A}[I]$ the subarrangement $\left\{A_{i} \mid i \in I\right\}$.

\subsection{Diagram-Preserving Maps}

Now let $\mathcal{A}=\left\{A_{1}, \ldots, A_{n}\right\}, \mathcal{B}=\left\{B_{1}, \ldots, B_{n}\right\}$ where each $A_{i}, B_{j}$ is a subcomplex of a finite regular cell complex for $1 \leq i, j \leq n$.

Definition 1.5 (Diagram-preserving maps) We call a map $f:\left|\mathcal{A}^{[n]}\right| \rightarrow\left|\mathcal{B}^{[n]}\right|$ to be diagram preserving if $f\left(\left|\mathcal{A}_{I}\right|\right) \subset\left|\mathcal{B}_{I}\right|$ for every $I \subset[n]$. (Notice that the above property is equivalent to $f\left(\left|A_{i}\right|\right) \subset\left|B_{i}\right|$ for every $i \in[n]$ but the previous property will be more convenient for us later when we extend the definition of diagrampreserving maps to homotopy co-limits (see Definition 3.3).) We say that two maps $f, g:\left|\mathcal{A}^{[n]}\right| \rightarrow\left|\mathcal{B}^{[n]}\right|$ are diagram homotopic if there exists a homotopy $h:\left|\mathcal{A}^{[n]}\right| \times$ $[0,1] \rightarrow\left|\mathcal{B}^{[n]}\right|$, such that $h(\cdot, 0)=f, h(\cdot, 1)=g$ and $h(\cdot, t)$ is diagram preserving for each $t \in[0,1]$.

More generally, we call a map $f: \mathbf{S}^{N}\left|\mathcal{A}^{[n]}\right| \rightarrow \mathbf{S}^{N}\left|\mathcal{B}^{[n]}\right|$ to be diagram preserving if $f\left(\mathbf{S}^{N}\left|\mathcal{A}_{I}\right|\right) \subset \mathbf{S}^{N}\left|\mathcal{B}_{I}\right|$ for every $I \subset[n]$. We say that two maps $f, g: \mathbf{S}^{N}\left|\mathcal{A}^{[n]}\right| \rightarrow$ $\mathbf{S}^{N}\left|\mathcal{B}^{[n]}\right|$ are diagram homotopic if there exists a homotopy $h: \mathbf{S}^{N}\left|\mathcal{A}^{[n]}\right| \times[0,1] \rightarrow$ $\mathbf{S}^{N}\left|\mathcal{B}^{[n]}\right|$ such that $h(\cdot, 0)=f, h(\cdot, 1)=g$ and $h(\cdot, t)$ is diagram preserving for each $t \in[0,1]$.

We say that $f:\left|\mathcal{A}^{[n]}\right| \rightarrow\left|\mathcal{B}^{[n]}\right|$ is a diagram-preserving homeomorphism if there exists a diagram-preserving inverse map $g:\left|\mathcal{B}^{[n]}\right| \rightarrow\left|\mathcal{A}^{[n]}\right|$ such that the induced maps $g \circ f:\left|\mathcal{A}^{[n]}\right| \rightarrow\left|\mathcal{A}^{[n]}\right|$ and $f \circ g:\left|\mathcal{B}^{[n]}\right| \rightarrow\left|\mathcal{B}^{[n]}\right|$ are $\operatorname{Id}_{\left|\mathcal{A}^{[n]}\right|}$ and $\operatorname{Id}_{\left|\mathcal{B}^{[n]}\right|}$, respectively.

We say that $f:\left|\mathcal{A}^{[n]}\right| \rightarrow\left|\mathcal{B}^{[n]}\right|$ is a diagram-preserving homotopy equivalence if there exists a diagram-preserving inverse map $g:\left|\mathcal{B}^{[n]}\right| \rightarrow\left|\mathcal{A}^{[n]}\right|$ such that the induced maps $g \circ f:\left|\mathcal{A}^{[n]}\right| \rightarrow\left|\mathcal{A}^{[n]}\right|$ and $f \circ g:\left|\mathcal{B}^{[n]}\right| \rightarrow\left|\mathcal{B}^{[n]}\right|$ are diagram homotopic to $\operatorname{Id}_{\left|\mathcal{A}^{[n]}\right|}$ and $\operatorname{Id}_{\mid \mathcal{B}}{ }^{[n]} \mid$, respectively. 
We say that an S-map $f \in\left\{\left|\mathcal{A}^{[n]}\right| ;\left|\mathcal{B}^{[n]}\right|\right\}$ is a diagram-preserving stable homotopy equivalence if it is represented by a diagram-preserving map

$$
\tilde{f}: \mathbf{S}^{N}\left|\mathcal{A}^{[n]}\right| \rightarrow \mathbf{S}^{N}\left|\mathcal{B}^{[n]}\right|
$$

such that there exists a diagram-preserving inverse map

$$
\tilde{g}: \mathbf{S}^{N}\left|\mathcal{B}^{[n]}\right| \rightarrow \mathbf{S}^{N}\left|\mathcal{A}^{[n]}\right|
$$

for which the induced maps

$$
\tilde{g} \circ \tilde{f}: \mathbf{S}^{N}\left|\mathcal{A}^{[n]}\right| \rightarrow \mathbf{S}^{N}\left|\mathcal{A}^{[n]}\right|,
$$

and

$$
\tilde{f} \circ \tilde{g}: \mathbf{S}^{N}\left|\mathcal{B}^{[n]}\right| \rightarrow \mathbf{S}^{N}\left|\mathcal{B}^{[n]}\right|
$$

are diagram homotopic to $\operatorname{Id}_{\mathbf{S}^{N}\left|\mathcal{A}^{[n]}\right|}$ and $\operatorname{Id}_{\mathbf{S}^{N}\left|\mathcal{B}^{[n]}\right|}$, respectively.

Translating these topological definitions into the language of arrangements, we say that:

Definition 1.6 (Topological type of an arrangement) Two arrangements $\mathcal{A}, \mathcal{B}$ are homeomorphic (respectively, homotopy equivalent, stable homotopy equivalent) if there exists a diagram-preserving homeomorphism (respectively, homotopy equivalence, stable homotopy equivalence) between them.

Remark 1.7 Note that, since two definable sets might be stable homotopy equivalent, without being homotopy equivalent (see [17, pp. 462]), and also homotopy equivalent without being homeomorphic, the notions of homeomorphism type, homotopy type and stable homotopy type are each strictly weaker than the previous one.

The main results of this paper can now be stated.

\subsection{Main Results}

Let $\mathcal{S}(\mathbb{R})$ be an o-minimal structure over $\mathbb{R}, T \subset \mathbb{R}^{k_{1}+k_{2}+\ell}$ a closed and bounded definable set, and let $\pi_{1}: \mathbb{R}^{k_{1}+k_{2}+\ell} \rightarrow \mathbb{R}^{k_{1}+k_{2}}$ (respectively, $\pi_{2}: \mathbb{R}^{k_{1}+k_{2}+\ell} \rightarrow \mathbb{R}^{\ell}$, $\pi_{3}: \mathbb{R}^{k_{1}+k_{2}} \rightarrow \mathbb{R}^{k_{2}}$ ) denote the projections onto the first $k_{1}+k_{2}$ (respectively, the last $\ell$, the last $\left.k_{2}\right)$ coordinates. For any collection $\mathcal{A}=\left\{A_{1}, \ldots, A_{n}\right\}$ of $\left(T, \pi_{1}, \pi_{2}\right)$ sets, and $\mathbf{z} \in \mathbb{R}^{k_{2}}$, we will denote by $\mathcal{A}_{\mathbf{z}}$ the collection of sets, $\left\{A_{1, \mathbf{z}}, \ldots, A_{n, \mathbf{z}}\right\}$, where $A_{i, \mathbf{z}}=A_{i} \cap \pi_{3}^{-1}(\mathbf{z}), 1 \leq i \leq n$.

A fundamental theorem in o-minimal geometry is Hardt's trivialization theorem (Theorem 4.2 here) which says that there exists a definable partition of $\mathbb{R}^{k_{2}}$ into a finite number of definable sets $\left\{T_{i}\right\}_{i \in I}$ such that for each $i \in I$, all fibers $\mathcal{A}_{\mathbf{z}}$ with $\mathbf{z} \in T_{i}$ are definably homeomorphic. A very natural question is to ask for an upper bound on the size of this partition (which will also give an upper bound on the number of homeomorphism types among the arrangements $\mathcal{A}_{\mathbf{z}}, \mathbf{z} \in \mathbb{R}^{k_{2}}$ ). 
Hardt's theorem is a corollary of the existence of cylindrical cell decompositions of definable sets proved in [11] (see also [6, 20]). When $\mathcal{A}$ is a $\left(T, \pi_{1}, \pi_{2}\right)$ family for some fixed definable set $T \subset \mathbb{R}^{k_{1}+k_{2}+\ell}$, with $\pi_{1}: \mathbb{R}^{k_{1}+k_{2}+\ell} \rightarrow \mathbb{R}^{k_{1}+k_{2}}$, $\pi_{2}: \mathbb{R}^{k_{1}+k_{2}+\ell} \rightarrow \mathbb{R}^{\ell}, \pi_{2}: \mathbb{R}^{k_{1}+k_{2}} \rightarrow \mathbb{R}^{k_{2}}$ the usual projections, and $\# \mathcal{A}=n$, the quantitative definable cylindrical cell decomposition theorem in [2] gives a doubly exponential (in $k_{1} k_{2}$ ) upper bound on the cardinality of $I$ and hence on the number of homeomorphism types among the arrangements $\mathcal{A}_{\mathbf{z}}, \mathbf{z} \in \mathbb{R}^{k_{2}}$. A tighter (say singly exponential) bound on the number of homeomorphism types of the fibers would be very interesting but is unknown at present. Note that we cannot hope for a bound that is better than singly exponential because the lower bounds on the number of topological types proved in [4] also applies in our situation.

In this paper we give tighter (singly exponential) upper bounds on the number of homotopy types occurring among the fibers $\mathcal{A}_{\mathbf{z}}, \mathbf{z} \in \mathbb{R}^{k_{2}}$. We prove the following theorems. The first theorem gives a bound on the number of stable homotopy types of the arrangements $\mathcal{A}_{\mathbf{z}}, \mathbf{z} \in \mathbb{R}^{k_{2}}$, while the second theorem gives a slightly worse bound for homotopy types.

Theorem 1.8 There exists a constant $C=C(T)>0$ such that for any collection $\mathcal{A}=\left\{A_{1}, \ldots, A_{n}\right\}$ of $\left(T, \pi_{1}, \pi_{2}\right)$-sets the number of distinct stable homotopy types amongst the arrangements $\mathcal{A}_{\mathbf{z}}, \mathbf{z} \in \mathbb{R}^{k_{2}}$ is bounded by

$$
C \cdot n^{\left(k_{1}+1\right) k_{2}} \text {. }
$$

If we replace stable homotopy type by homotopy type, we obtain a slightly weaker bound.

Theorem 1.9 There exists a constant $C=C(T)>0$ such that for any collection $\mathcal{A}=$ $\left\{A_{1}, \ldots, A_{n}\right\}$ of $\left(T, \pi_{1}, \pi_{2}\right)$-sets the number of distinct homotopy types occurring among the arrangements $\mathcal{A}_{\mathbf{z}}, \mathbf{z} \in \mathbb{R}^{k_{2}}$ is bounded by

$$
C \cdot n^{\left(k_{1}+3\right) k_{2}} \text {. }
$$

\section{Background}

In this section we describe some prior work in the area of bounding the number of homotopy types of fibers of a definable map and their connections with the results presented in this paper.

We begin with a definition.

Definition 2.1 ( $\mathcal{A}$-sets) Let $\mathcal{A}=\left\{A_{1}, \ldots, A_{n}\right\}$, such that each $A_{i} \subset \mathbb{R}^{k}$ is a $\left(T, \pi_{1}, \pi_{2}\right)$-set. For $I \subset\{1, \ldots, n\}$, we let $\mathcal{A}(I)$ denote the set

$$
\bigcap_{i \in I \subset[n]} A_{i} \cap \bigcap_{j \in[n] \backslash I}\left(\mathbb{R}^{k} \backslash A_{j}\right)
$$

and we will call such a set to be a basic $\mathcal{A}$-set. We will denote by $\mathcal{C}(\mathcal{A})$ the set of nonempty connected components of all basic $\mathcal{A}$-sets. 
We will call definable subsets $S \subset \mathbb{R}^{k}$ defined by a Boolean formula whose atoms are of the form, $x \in A_{i}, 1 \leq i \leq n$, an $\mathcal{A}$-set. An $\mathcal{A}$-set is thus a union of basic $\mathcal{A}$-sets. If $T$ is closed, and the Boolean formula defining $S$ has no negations, then $S$ is closed by definition (since each $A_{i}$ is closed) and we call such a set an $\mathcal{A}$-closed set.

Moreover, if $V$ is any closed definable subset of $\mathbb{R}^{k}$, and $S$ is an $\mathcal{A}$-set (respectively, $\mathcal{A}$-closed set), then we will call $S \cap V$ to be an $(\mathcal{A}, V)$-set (respectively, $(\mathcal{A}, V)$-closed set).

\subsection{Bounds on the Betti Numbers of Admissible Sets}

The problem of bounding the Betti numbers of $\mathcal{A}$-sets is investigated in [2], where several results known in the semi-algebraic and semi-Pfaffian case are extended to this general setting. In particular, we will need the following theorem proved there.

Theorem $2.2[2]$ Let $\mathcal{S}(\mathbb{R})$ be an o-minimal structure over $\mathbb{R}$ and let $T \subset \mathbb{R}^{k+\ell}$ be a closed definable set. Then, there exists a constant $C=C(T)>0$ depending only on $T$ such that for any arrangement $\mathcal{A}=\left\{A_{1}, \ldots, A_{n}\right\}$ of $\left(T, \pi_{1}, \pi_{2}\right)$-sets of $\mathbb{R}^{k}$ the following holds.

For every $i, 0 \leq i \leq k$,

$$
\sum_{D \in \mathcal{C}(\mathcal{A})} b_{i}(D) \leq C \cdot n^{k-i}
$$

Remark 2.3 The main intuition behind the bound in Theorem 2.2 (as well as similar results in the semi-algebraic and semi-Pfaffian settings) is that the homotopy type (or at least the Betti numbers) of a definable set in $\mathbb{R}^{k}$ defined in terms of $n$ sets belonging to some fixed definable family, depend only on the interaction of these sets at most $k+1$ at a time. This is reminiscent of Helly's theorem in convexity theory (see [7]) but in a homotopical setting. This observation is also used to give an efficient algorithm for computing the Betti numbers of arrangements (see [3, Sect. 8]). However, the proof of Theorem 2.2 in [2] (as well as the proofs of similar results in the semialgebraic [5] and semi-Pfaffian settings [10]) depends on an argument involving the Mayer-Vietoris sequence for homology, and does not require more detailed information about homotopy types. In Sect. 3, we make the above intuition mathematically precise.

We prove two theorems (Theorems 3.6 and 3.7) and these auxiliary results are the keys to proving the main results of this paper (Theorems 1.8 and 1.9). Moreover, these auxiliary results could also be of independent interest in the quantitative study of arrangements.

\subsection{Homotopy Types of the Fibers of a Semi-algebraic Map}

Theorem 2.2 gives tight bounds on the topological complexity of an $\mathcal{A}$-set in terms of the cardinality of $\mathcal{A}$, assuming that the sets in $\mathcal{A}$ belong to some fixed definable family. A problem closely related to the problem we consider in this paper is to bound the number of topological types of the fibers of a projection restricted to an arbitrary $\mathcal{A}$-set. 
More precisely, let $S \subset \mathbb{R}^{k_{1}+k_{2}}$ be a set definable in an o-minimal structure over the reals (see [20]) and let $\pi: \mathbb{R}^{k_{1}+k_{2}} \rightarrow \mathbb{R}^{k_{2}}$ denote the projection map on the last $k_{2}$ coordinates. We consider the fibers, $S_{\mathbf{z}}=\pi^{-1}(\mathbf{z}) \cap S$ for different $\mathbf{z}$ in $\mathbb{R}^{k_{2}}$. Hardt's trivialization theorem, (Theorem 4.2) shows that there exists a definable partition of $\mathbb{R}^{k_{2}}$ into a finite number of definable sets $\left\{T_{i}\right\}_{i \in I}$ such that for each $i \in I$ and any point $\mathbf{z}_{i} \in T_{i}, \pi^{-1}\left(T_{i}\right) \cap S$ is definably homeomorphic to $S_{\mathbf{z}_{i}} \times T_{i}$ by a fiberpreserving homeomorphism. In particular, for each $i \in I$, all fibers $S_{\mathbf{z}}$ with $\mathbf{z} \in T_{i}$ are definably homeomorphic.

In case $S$ is an $\mathcal{A}$-set, with $\mathcal{A}$ a $\left(T, \pi_{1}, \pi_{2}\right)$-family for some fixed definable set $T \subset \mathbb{R}^{k_{1}+k_{2}+\ell}$, with $\pi_{1}: \mathbb{R}^{k_{1}+k_{2}+\ell} \rightarrow \mathbb{R}^{k_{1}+k_{2}}, \pi_{2}: \mathbb{R}^{k_{1}+k_{2}+\ell} \rightarrow \mathbb{R}^{\ell}, \pi_{2}: \mathbb{R}^{k_{1}+k_{2}} \rightarrow$ $\mathbb{R}^{k_{2}}$, the usual projections, and $\# \mathcal{A}=n$, the quantitative definable cylindrical cell decomposition theorem in [2] gives a doubly exponential (in $k_{1} k_{2}$ ) upper bound on the cardinality of $I$ and hence on the number of homeomorphism types of the fibers of the map $\left.\pi_{3}\right|_{S}$. A tighter (say singly exponential) bound on the number of homeomorphism types of the fibers would be very interesting but is unknown at present.

Recently, the problem of obtaining a tight bound on the number of topological types of the fibers of a definable map for semi-algebraic and semi-Pfaffian sets was considered in [4], and it was shown that the number of distinct homotopy types of the fibers of such a map can be bounded (in terms of the format of the formula defining the set) by a function singly exponential in $k_{1} k_{2}$. In particular, the combinatorial part of the bound is also singly exponential. A more precise statement in the case of semialgebraic sets is the following theorem which appears in [4].

Theorem 2.4 [4] Let $\mathcal{P} \subset \mathbb{R}\left[X_{1}, \ldots, X_{k_{1}}, Y_{1}, \ldots, Y_{k_{2}}\right]$, with $\operatorname{deg}(P) \leq d$ for each $P \in \mathcal{P}$ and cardinality $\# \mathcal{P}=n$. Then, for any fixed $\mathcal{P}$-semi-algebraic set $S$ the number of different homotopy types of fibers $\pi^{-1}(\mathbf{y}) \cap S$ for various $\mathbf{y} \in \pi(S)$ is bounded by

$$
\left(2^{k_{1}} n k_{2} d\right)^{O\left(k_{1} k_{2}\right)}
$$

Remark 2.5 The proof of Theorem 2.4, however, has the drawback that it relies on techniques involving perturbations of the original polynomials in order to put them in general position, as well as on Thom's Isotopy Theorem, and as such does not extend easily to the o-minimal setting. The main results of this paper (see Theorems 1.8 and 1.9) extend the combinatorial part of Theorem 2.4 to the more general o-minimal category.

Remark 2.6 Even though the formulation of Theorem 2.4 seems a little different from the main theorems of this paper (Theorems 1.8 and 1.9), they are in fact closely related. In fact, as a consequence of Theorem 1.9 we obtain bounds on the number of homotopy types of the fibers of $S$ for any fixed $\mathcal{A}$-set $S$, analogous to the one in Theorem 2.4.

More precisely we have:

Theorem 2.7 Let $\mathcal{S}(\mathbb{R})$ be an o-minimal structure over $\mathbb{R}$, and $T \subset \mathbb{R}^{k_{1}+k_{2}+\ell}$ a closed and bounded definable set, and $\pi_{1}: \mathbb{R}^{k_{1}+k_{2}+\ell} \rightarrow \mathbb{R}^{k_{1}+k_{2}}, \pi_{2}: \mathbb{R}^{k_{1}+k_{2}+\ell} \rightarrow$ $\mathbb{R}^{\ell}$, and $\pi_{3}: \mathbb{R}^{k_{1}+k_{2}} \rightarrow \mathbb{R}^{k_{2}}$ the projection maps. Then, there exists a constant 
$C=C(T)>0$, such that for any collection $\mathcal{A}=\left\{A_{1}, \ldots, A_{n}\right\}$ of $\left(T, \pi_{1}, \pi_{2}\right)$-sets, for any fixed $\mathcal{A}$-set $S$ the number of distinct homotopy types of fibers $\pi_{3}^{-1}(\mathbf{z}) \cap S$ for various $\mathbf{z} \in \pi_{3}(S)$ is bounded by

$$
C \cdot n^{\left(k_{1}+3\right) k_{2}} \text {. }
$$

A similar result with a bound of $C \cdot n^{\left(k_{1}+1\right) k_{2}}$ holds for stable homotopy types as well.

\section{A Topological Comparison Theorem}

As noted previously, the main underlying idea behind our proof of Theorem 1.8 is that the homotopy type of an $\mathcal{A}$-set in $\mathbb{R}^{k}$ depends only on the interaction of sets in $\mathcal{A}$ at most $(k+1)$ at a time. In this section we make this idea precise.

We show that in case $\mathcal{A}=\left\{A_{1}, \ldots, A_{n}\right\}$, with each $A_{i}$ a definable, closed and bounded subset of $\mathbb{R}^{k}$, the homotopy type of any $\mathcal{A}$-closed set is determined by a certain subcomplex of the homotopy co-limit of the diagram of $\mathcal{A}$. The crucial fact here is that this subcomplex depends only on the intersections of the sets in $\mathcal{A}$ at most $k+1$ at a time.

In order to avoid technical difficulties, we restrict ourselves to the category of finite, regular cell complexes (see [25] for the definition of a regular cell complex). The setting of finite, regular cell complexes suffices for us, since it is well known that closed and bounded definable sets in any o-minimal structure are finitely triangulable and, hence, are homeomorphic to regular cell complexes.

\subsection{Topological Preliminaries}

Let $\mathcal{A}=\left\{A_{1}, \ldots, A_{n}\right\}$, where each $A_{i}$ is a subcomplex of a finite regular cell complex. We now define the homotopy co-limit of the diagram of $\mathcal{A}$.

\subsubsection{Homotopy Co-limits}

Let $\Delta_{[n]}$ denote the standard simplex of dimension $n-1$ with vertices in $[n]$ (and by $\left|\Delta_{[n]}\right|$ the corresponding closed geometric simplex). For $I \subset[n]$, we denote by $\Delta_{I}$ the $(\# I-1)$-dimensional face of $\Delta_{[n]}$ corresponding to $I$.

The homotopy co-limit, $\operatorname{hocolim}(\mathcal{A})$, is a $\mathrm{CW}$-complex defined as follows.

Definition 3.1 (Homotopy co-limit)

$$
\operatorname{hocolim}(\mathcal{A})=\coprod_{I \subset[n]} \Delta_{I} \times \mathcal{A}_{I} / \sim,
$$

where the equivalence relation $\sim$ is defined as follows.

For $I \subset J \subset[n]$, let $s_{I, J}:\left|\Delta_{I}\right| \hookrightarrow\left|\Delta_{J}\right|$ denote the inclusion map of the face $\left|\Delta_{I}\right|$ in $\left|\Delta_{J}\right|$, and let $i_{I, J}:\left|\mathcal{A}_{J}\right| \hookrightarrow\left|\mathcal{A}_{I}\right|$ denote the inclusion map of $\left|\mathcal{A}_{J}\right|$ in $\left|\mathcal{A}_{I}\right|$.

Given $(\mathbf{s}, \mathbf{x}) \in\left|\Delta_{I}\right| \times\left|\mathcal{A}_{I}\right|$ and $(\mathbf{t}, \mathbf{y}) \in\left|\Delta_{J}\right| \times\left|\mathcal{A}_{J}\right|$ with $I \subset J$, then $(\mathbf{s}, \mathbf{x}) \sim(\mathbf{t}, \mathbf{y})$ if and only if $\mathbf{t}=s_{I, J}(\mathbf{s})$ and $\mathbf{x}=i_{I, J}(\mathbf{y})$. 
Note that there exist two natural maps

$$
\begin{aligned}
& f_{\mathcal{A}}:|\operatorname{hocolim}(\mathcal{A})| \rightarrow\left|\mathcal{A}^{[n]}\right|, \\
& g_{\mathcal{A}}:|\operatorname{hocolim}(\mathcal{A})| \rightarrow\left|\Delta_{[n]}\right|
\end{aligned}
$$

defined by

$$
f_{\mathcal{A}}(\mathbf{s}, \mathbf{x})=\mathbf{s},
$$

and

$$
g_{\mathcal{A}}(\mathbf{s}, \mathbf{x})=\mathbf{x},
$$

where $(\mathbf{s}, \mathbf{x}) \in\left|\Delta_{I_{c}}\right| \times c, c$ is a cell in $\mathcal{A}^{[n]}$ and $I_{c}=\left\{i \in[n] \mid c \in A_{i}\right\}$.

Notice that we have

$$
|\operatorname{hocolim}(\mathcal{A})|=\bigcup_{I \subset[n]}\left|\Delta_{I}\right| \times\left|\mathcal{A}_{I}\right| \subset \bigcup_{I \subset[n]}\left|\Delta_{I}\right| \times \mathcal{A}^{[n]} .
$$

Definition 3.2 (Truncated homotopy co-limits) For any $m, 0 \leq m \leq n$, we will denote by $\operatorname{hocolim}_{m}(\mathcal{A})$ the subcomplex of $\operatorname{hocolim}(\mathcal{A})$ defined by

$$
\operatorname{hocolim}_{m}(\mathcal{A})=g_{\mathcal{A}}^{-1}\left(\operatorname{sk}_{m}\left(\Delta_{[n]}\right)\right)
$$

Definition 3.3 (Diagram-preserving maps between homotopy co-limits) Replacing in Definition $1.5,\left|\mathcal{A}^{[n]}\right|$ and $\left|\mathcal{B}^{[n]}\right|$, by $|\operatorname{hocolim}(\mathcal{A})|$ and $|\operatorname{hocolim}(\mathcal{B})|$, respectively, as well as $\left|\mathcal{A}_{I}\right|$ and $\left|\mathcal{B}_{I}\right|$ by $f_{\mathcal{A}}^{-1}\left(\left|\mathcal{A}_{I}\right|\right)$ and $f_{\mathcal{B}}^{-1}\left(\left|\mathcal{B}_{I}\right|\right)$, respectively, we get definitions of diagram-preserving homotopy equivalences and stable homotopy equivalences between $|\operatorname{hocolim}(\mathcal{A})|$ and $|\operatorname{hocolim}(B)|$, and more generally for any $m \geq 0$, between $\left|\operatorname{hocolim}_{m}(\mathcal{A})\right|$ and $\left|\operatorname{hocolim}_{m}(\mathcal{B})\right|$.

Definition 3.4 We say that $\mathcal{A} \approx_{m} \mathcal{B}$ if there exists a diagram-preserving homotopy equivalence

$$
\phi:\left|\operatorname{hocolim}_{m}(\mathcal{A})\right| \rightarrow\left|\operatorname{hocolim}_{m}(\mathcal{B})\right| .
$$

We say that $\mathcal{A} \sim_{m} \mathcal{B}$, if there exists a diagram-preserving stable homotopy equivalence $\phi \in\left\{\operatorname{hocolim}_{m}(\mathcal{A}) ; \operatorname{hocolim}_{m}(\mathcal{B})\right\}$, represented by

$$
\tilde{\phi}: \mathbf{S}^{N}\left|\operatorname{hocolim}_{m}(\mathcal{A})\right| \rightarrow \mathbf{S}^{N}\left|\operatorname{hocolim}_{m}(\mathcal{B})\right|,
$$

for some $N>0$.

Remark 3.5 Note that in the above definition the map $\phi$ need not be induced by a diagram-preserving map $\phi: \mathcal{A}^{[n]} \rightarrow \mathcal{B}^{[n]}$ (respectively, $\tilde{\phi}: \mathbf{S}^{N}\left|\operatorname{hocolim}_{m}(\mathcal{A})\right| \rightarrow$ $\mathbf{S}^{N} \mid$ hocolim $\left._{m}(\mathcal{B}) \mid\right)$. Indeed if it was the case then the proofs of Theorems 3.6 and 3.7 below would be simplified considerably.

The two following theorems which are proved in Sect. 3.2 are the crucial topological ingredients in the proofs of our main results. 
Theorem 3.6 Let $\mathcal{A}=\left\{A_{1}, \ldots, A_{n}\right\}, \mathcal{B}=\left\{B_{1}, \ldots, B_{n}\right\}$ be two families of subcomplexes of a finite regular cell complex, such that:

(1) $\mathrm{H}_{i}\left(\left|\mathcal{A}^{[n]}\right|, \mathbb{Z}\right), \mathrm{H}_{i}\left(\left|\mathcal{B}^{[n]}\right|, \mathbb{Z}\right)=0$, for all $i \geq k$, and

(2) $\mathcal{A} \sim_{k} \mathcal{B}$

Then, $\mathcal{A}$ and $\mathcal{B}$ are stable homotopy equivalent.

Theorem 3.7 Let $\mathcal{A}=\left\{A_{1}, \ldots, A_{n}\right\}, \mathcal{B}=\left\{B_{1}, \ldots, B_{n}\right\}$ be two families of subcomplexes of a finite regular cell complex, such that:

(1) $\operatorname{dim}\left(A_{i}\right), \operatorname{dim}\left(B_{i}\right) \leq k$, for $1 \leq i \leq n$, and

(2) $\mathcal{A} \approx_{k+2} \mathcal{B}$.

Then, $\mathcal{A}$ and $\mathcal{B}$ are homotopy equivalent.

We now state two corollaries of Theorems 3.6 and 3.7 which might be of interest.

Given a Boolean formula $\theta\left(T_{1}, \ldots, T_{n}\right)$ containing no negations and a family of subcomplexes $\mathcal{A}=\left\{A_{1}, \ldots, A_{n}\right\}$ of a finite regular cell complex, we will denote by $\mathcal{A}_{\theta}$ the subcomplex defined by the formula, $\theta_{\mathcal{A}}$, which is obtained from $\theta$ by replacing in $\theta$ the atom $T_{i}$ by $A_{i}$ for each $i \in[n]$, and replacing each $\wedge$ (respectively, $\vee$ ) by $\cap$ (respectively, $\cup$ ).

Corollary 3.8 Let $\mathcal{A}=\left\{A_{1}, \ldots, A_{n}\right\}, \mathcal{B}=\left\{B_{1}, \ldots, B_{n}\right\}$ be two families of subcomplexes of a finite regular cell complex, satisfying the same conditions as in Theorem 3.6. Let $\theta\left(T_{1}, \ldots, T_{n}\right)$ be a Boolean formula without negations. Then, $\left|\mathcal{A}_{\theta}\right|$ and $\left|\mathcal{B}_{\theta}\right|$ are stable homotopy equivalent.

Corollary 3.9 Let $\mathcal{A}=\left\{A_{1}, \ldots, A_{n}\right\}, \mathcal{B}=\left\{B_{1}, \ldots, B_{n}\right\}$ be two families of subcomplexes of a finite regular cell complex, satisfying the same conditions as in Theorem 3.7. Let $\theta\left(T_{1}, \ldots, T_{n}\right)$ be a Boolean formula without negations. Then, $\left|\mathcal{A}_{\theta}\right|$ and $\left|\mathcal{B}_{\theta}\right|$ are homotopy equivalent.

\subsection{Proofs of Theorems 3.6 and 3.7}

Let $\mathcal{A}$ and $\mathcal{B}$ as in Theorem 3.6.

We need a preliminary lemma.

Lemma 3.10 $\left|\mathcal{A}^{[n]}\right|$ is diagram-preserving homotopy equivalent to $|\operatorname{hocolim}(\mathcal{A})|$.

Proof Consider the map

$$
f_{\mathcal{A}}:|\operatorname{hocolim}(\mathcal{A})| \rightarrow\left|\mathcal{A}^{[n]}\right|
$$

defined in (3.1).

Clearly, if $\mathbf{x} \in c, f_{\mathcal{A}}^{-1}(c)=\left|\Delta_{I_{c}}\right| \times c$. Now applying Smale's version of the Vietoris-Begle Theorem [16] we obtain that $f_{\mathcal{A}}$ is a homotopy equivalence. Clearly, 
$f_{\mathcal{A}}$ is diagram preserving. Moreover, (see, for instance, the proof of Theorem 6 in [16]) there exists an cellular inverse map

$$
h_{\mathcal{A}}:\left|\mathcal{A}^{[n]}\right| \rightarrow|\operatorname{hocolim}(\mathcal{A})|
$$

such that $f_{\mathcal{A}} \circ h_{\mathcal{A}}$ is diagram preserving, and is a homotopy inverse of $f_{\mathcal{A}}$.

We can now prove Theorems 3.6 and 3.7.

Proof of Theorem 3.6 Let $h_{\mathcal{A}}:\left|\mathcal{A}^{[n]}\right| \rightarrow|\operatorname{hocolim}(\mathcal{A})|$ be a diagram-preserving homotopy equivalence known to exist by Lemma 3.10. Since $h_{\mathcal{A}}$ is cellular, and $\operatorname{dim}\left|\mathcal{A}^{[n]}\right| \leq k$, its image is contained in $\operatorname{hocolim}_{k}(\mathcal{A})$ since by definition (see (3.3))

$$
\operatorname{sk}_{k}(\operatorname{hocolim}(\mathcal{A})) \subset \operatorname{hocolim}_{k}(\mathcal{A}) .
$$

We will denote by $h_{\mathcal{A}, \mathcal{B}}: \mathbf{S}^{N}\left|\operatorname{hocolim}_{k}(\mathcal{A})\right| \rightarrow \mathbf{S}^{N}\left|\operatorname{hocolim}_{k}(\mathcal{B})\right|$ a map representing a diagram-preserving stable homotopy equivalence known to exist by hypothesis (which we assume to be cellular).

Let $i_{\mathcal{B}, k}: \mathbf{S}^{N}\left|\operatorname{hocolim}_{k}(\mathcal{B})\right| \hookrightarrow \mathbf{S}^{N}|\operatorname{hocolim}(\mathcal{B})|$ denote the inclusion map. The map $i_{\mathcal{B}, k}$ induces isomorphisms

$$
\left(i_{\mathcal{B}, k}\right)_{*}: \mathrm{H}_{j}\left(\operatorname{hocolim}_{k}(\mathcal{B}), \mathbb{Z}\right) \rightarrow \mathrm{H}_{j}(\operatorname{hocolim}(\mathcal{B}), \mathbb{Z})
$$

for $0 \leq j \leq k-1$.

Consequently, the map $f_{\mathcal{B}} \circ i_{\mathcal{B}, k}$ induces isomorphisms

$$
\left(f_{\mathcal{B}} \circ i_{\mathcal{B}, k}\right)_{*}: \mathrm{H}_{j}\left(\operatorname{hocolim}_{k}(\mathcal{B}), \mathbb{Z}\right) \rightarrow \mathrm{H}_{j}\left(\mathcal{B}^{[n]}, \mathbb{Z}\right)
$$

for $0 \leq j \leq k-1$.

Composing the maps, $\mathbf{S}^{N} h_{\mathcal{A}}, h_{\mathcal{A B}}, i_{\mathcal{B}, k}, \mathbf{S}^{N} f_{\mathcal{B}}$ we obtain that the map,

$$
\mathbf{S}^{N} f_{\mathcal{B}} \circ i_{\mathcal{B}, k} \circ h_{\mathcal{A B}} \circ \mathbf{S}^{N} h_{\mathcal{A}}: \mathbf{S}^{N}\left|\mathcal{A}^{[n]}\right| \rightarrow \mathbf{S}^{N}\left|\mathcal{B}^{[n]}\right|
$$

induces isomorphisms

$$
\left(\mathbf{S}^{N} f_{\mathcal{B}} \circ i_{\mathcal{B}, k} \circ h_{\mathcal{A}, \mathcal{B}, k} \circ \mathbf{S}^{N} h_{\mathcal{A}}\right)_{*}: \mathrm{H}_{j}\left(\left|\mathcal{A}^{[n]}\right|, \mathbb{Z}\right) \rightarrow \mathrm{H}_{j}\left(\left|\mathcal{B}^{[n]}\right|, \mathbb{Z}\right)
$$

for all $j \geq 0$.

Moreover, the map $\mathbf{S}^{N} f_{\mathcal{B}} \circ i_{\mathcal{B}, k} \circ h_{\mathcal{A B}} \circ \mathbf{S}^{N} h_{\mathcal{A}}$ is diagram preserving since each constituent of the composition is diagram preserving. It now follows from Theorem 1.4 that the S-map represented by

$$
\phi=\mathbf{S}^{N} f_{\mathcal{B}} \circ i_{\mathcal{B}, k} \circ h_{\mathcal{A B}} \circ \mathbf{S}^{N} h_{\mathcal{A}}: \mathbf{S}^{N}\left|\mathcal{A}^{[n]}\right| \rightarrow \mathbf{S}^{N}\left|\mathcal{B}^{[n]}\right|,
$$

is a diagram-preserving stable homotopy equivalence.

Before proving Theorem 3.7 we first need to recall a few basic facts from homotopy theory. 
Definition 3.11 ( $k$-equivalence) A map $f: X \rightarrow Y$ between two regular cell complex is called a $k$-equivalence if the induced homomorphism

$$
f_{*}: \pi_{i}(X) \rightarrow \pi_{i}(Y)
$$

is an isomorphism for all $0 \leq i<k$, and an epimorphism for $i=k$, and we say that $X$ is $k$-equivalent to $Y$. (Note that $k$-equivalence is not an equivalence relation).

We also need the following well-known fact from algebraic topology.

Proposition 3.12 Let $X, Y$ be finite regular cell complexes with

$$
\operatorname{dim}(X)<k, \operatorname{dim}(Y) \leq k,
$$

and $f: X \rightarrow Y$ a k-equivalence. Then, $f$ is a homotopy equivalence between $X$ and $Y$.

Proof See [24, pp. 69].

Proof of Theorem 3.7 The proof is along the same lines as that of the proof of Theorem 3.6. Let $h_{\mathcal{A}}:\left|\mathcal{A}^{[n]}\right| \rightarrow|\operatorname{hocolim}(\mathcal{A})|$ be a diagram-preserving homotopy equivalence known to exist by Lemma 3.10. By the same argument as before, its image is contained in $\left|\operatorname{hocolim}_{k+2}(\mathcal{A})\right|$.

We will denote by $h_{\mathcal{A}, \mathcal{B}}:\left|\operatorname{hocolim}_{k+2}(\mathcal{A})\right| \rightarrow\left|\operatorname{hocolim}_{k+2}(\mathcal{B})\right|$ a diagrampreserving homotopy equivalence known to exist by hypothesis.

Let $i_{\mathcal{B}, k+2}:\left|\operatorname{hocolim}_{k+2}(\mathcal{B})\right| \hookrightarrow|\operatorname{hocolim}(\mathcal{B})|$ denote the inclusion map. The map $i_{\mathcal{B}, k+2}$ induces isomorphisms

$$
\left(i_{\mathcal{B}, k+2}\right)_{*}: \pi_{j}\left(\operatorname{hocolim}_{k+2}(\mathcal{B})\right) \rightarrow \pi_{j}(\operatorname{hocolim}(\mathcal{B}))
$$

for $0 \leq j \leq k+1$. This is a consequence of the exactness of the homotopy sequence of the pair $\left(\operatorname{hocolim}(\mathcal{B}), \operatorname{hocolim}_{k+2}(\mathcal{B})\right)($ see [17]).

Consequently, the map $f_{\mathcal{B}} \circ i_{\mathcal{B}, k}$ induces isomorphisms

$$
\left(f_{\mathcal{B}} \circ i_{\mathcal{B}, k}\right)_{*}: \pi_{j}\left(\operatorname{hocolim}_{k+2}(\mathcal{B})\right) \rightarrow \pi_{j}\left(\mathcal{B}^{[n]}\right)
$$

for $0 \leq j \leq k+1$.

Composing the maps, $h_{\mathcal{A}}, h_{\mathcal{A B}}, i_{\mathcal{B}, k+2}, f_{\mathcal{B}}$ we obtain that the map

$$
f_{\mathcal{B}} \circ i_{\mathcal{B}, k} \circ h_{\mathcal{A B}} \circ h_{\mathcal{A}}:\left|\mathcal{A}^{[n]}\right| \rightarrow\left|\mathcal{B}^{[n]}\right|
$$

induces isomorphisms

$$
\left(f_{\mathcal{B}} \circ i_{\mathcal{B}, k} \circ h_{\mathcal{A}, \mathcal{B}, k} \circ h_{\mathcal{A}}\right)_{*}: \pi_{j}\left(\mathcal{A}^{[n]}\right) \rightarrow \pi_{j}\left(\mathcal{B}^{[n]}\right)
$$

for $0 \leq j \leq k+1$. 
Moreover, the map $f_{\mathcal{B}} \circ i_{\mathcal{B}, k} \circ h_{\mathcal{A B}} \circ h_{\mathcal{A}}$ is diagram preserving since each constituent of the composition is diagram preserving. It now follows from Proposition 3.12 that the map

$$
\phi=f_{\mathcal{B}} \circ i_{\mathcal{B}, k} \circ h_{\mathcal{A B}} \circ h_{\mathcal{A}}:\left|\mathcal{A}^{[n]}\right| \rightarrow\left|\mathcal{B}^{[n]}\right|
$$

is a diagram-preserving homotopy equivalence.

Proof of Corollary 3.8 First note that since the formula $\theta$ does not contain negations, writing $\theta$ as a disjunction of conjunctions, there exists $\Sigma \subset 2^{[n]}$ such that $\mathcal{A}_{\theta}=\bigcup_{I \in \Sigma} \mathcal{A}_{I}$ (respectively, $\mathcal{B}_{\theta}=\bigcup_{I \in \Sigma} \mathcal{B}_{I}$ ). Let $\mathcal{A}^{\prime}=\left\{\mathcal{A}_{I} \mid I \in \Sigma\right\}$ (respectively, $\left.\mathcal{B}^{\prime}=\left\{\mathcal{B}_{I} \mid I \in \Sigma\right\}\right)$. It follows from the hypothesis that

$$
\mathcal{A}^{\prime} \sim_{k} \mathcal{B}^{\prime}
$$

Now apply Theorem 3.6.

Proof of Corollary 3.9 The proof is similar to that of Corollary 3.8 using Theorem 3.7 in place of Theorem 3.6 and is omitted.

\section{Proofs of the Main Theorems}

\subsection{Summary of the Main Ideas}

We first summarize the main ideas underlying the proof of Theorem 1.8. The proof of Theorem 1.9 is similar and differs only in technical details. Let $\mathcal{A}=\left\{A_{1}, \ldots, \mathcal{A}_{n}\right\}$ be a $\left(T, \pi_{1}, \pi_{2}\right)$ - arrangement in $\mathbb{R}^{k_{1}+k_{2}}$. Using Proposition 4.7 , we obtain a definable partition, $\left\{C_{\alpha}\right\}_{\alpha \in I}$ (say) of $\mathbb{R}^{k_{2}}$, into connected locally closed definable sets $C_{\alpha} \subset \mathbb{R}^{k_{2}}$, with the property that as $\mathbf{z}$ varies over $C_{\alpha}$, we get for each $I \subset[n]$ with $\# I \leq k_{1}+1$ isomorphic (and continuously varying) triangulations of the subarrangement $\mathcal{A}[I]$. Moreover, these triangulations are downward compatible in the sense that the restriction to $\mathcal{A}[J]$ of the triangulation of $\mathcal{A}[I]$, refines that of $\mathcal{A}[J]$ for each $J \subset I$ (cf. Proposition 4.7). These facts allow us to prove that for any $z_{1}, z_{2} \in C_{\alpha}$ the truncated homotopy co-limits $\left|\operatorname{hocolim} k_{1}\left(\mathcal{A}_{z_{1}}\right)\right|$ and $\left|\operatorname{hocolim}_{k_{1}}\left(\mathcal{A}_{z_{2}}\right)\right|$ are homotopy equivalent by a diagram-preserving homotopy equivalence. More precisely, we first prove that the thickened homotopy co-limits $\mid$ hocolim $k_{1}^{+}\left(\mathcal{A}_{z_{1}}, \bar{\varepsilon}\right) \mid$ and $\mid$ hocolim $_{k_{1}}^{+}\left(\mathcal{A}_{z_{2}}, \bar{\varepsilon}\right) \mid$ are homeomorphic, and then use Proposition 4.8 to deduce that $\mid$ hocolim $_{k_{1}}\left(\mathcal{A}_{z_{1}}\right) \mid$ and $\left|\operatorname{hocolim}_{k_{1}}\left(\mathcal{A}_{z_{2}}\right)\right|$ are homotopy equivalent. Theorem 3.6 then implies that $\mathcal{A}_{z_{1}}$ is stable homotopy equivalent to $\mathcal{A}_{\mathbf{z}_{2}}$ by a diagram-preserving stable homotopy equivalence. It remains to bound the number of elements in the partition $\left\{C_{\alpha}\right\}_{\alpha \in I}$. We use Theorem 2.2 to obtain a bound of $C \cdot n^{\left(k_{1}+1\right) k_{2}}$ on this number, where $C$ is a constant which depends only on $T$.

In order to prove Theorem 1.8 we recall a few results from o-minimal geometry.

We first note an elementary property of families of admissible sets (see [2] for a proof). 
Observation 4.1 Suppose that $T_{1}, \ldots, T_{m} \subset \mathbb{R}^{k+\ell}$ are definable sets, $\pi_{1}: \mathbb{R}^{k+\ell} \rightarrow$ $\mathbb{R}^{k}$ and $\pi_{2}: \mathbb{R}^{k+\ell} \rightarrow \mathbb{R}^{\ell}$ the two projections. Then, there exists a definable subset $T^{\prime} \subset \mathbb{R}^{k+\ell+m}$ depending only on $T_{1}, \ldots, T_{m}$, such that for any collection of $\left(T_{i}, \pi_{1}, \pi_{2}\right)$ families $\mathcal{A}_{i}, 1 \leq i \leq m$, the union $\bigcup_{i=1}^{m} \mathcal{A}_{i}$ is a $\left(T^{\prime}, \pi_{1}^{\prime}, \pi_{2}^{\prime}\right)$-family, where $\pi_{1}^{\prime}: \mathbb{R}^{k+m+\ell} \rightarrow \mathbb{R}^{k}$ and $\pi_{2}^{\prime}: \mathbb{R}^{k+\ell+m} \rightarrow \mathbb{R}^{\ell+m}$ are the projections onto the first $k$, and the last $\ell+m$ coordinates, respectively.

\subsection{Hardt's Triviality for Definable Sets}

One important technical tool will be the following o-minimal version of Hardt's triviality theorem.

Let $X \subset \mathbb{R}^{k} \times \mathbb{R}^{\ell}$ and $A \subset \mathbb{R}^{k}$ be definable subsets of $\mathbb{R}^{k} \times \mathbb{R}^{\ell}$ and $\mathbb{R}^{\ell}$, respectively, and let $\pi: X \rightarrow \mathbb{R}^{\ell}$ denote the projection map on the last $\ell$ coordinates.

We say that $X$ is definably trivial over $A$ if there exists a definable set $F$ and a definable homeomorphism

$$
h: F \times A \rightarrow X \cap \pi^{-1}(A),
$$

such that the following diagram commutes.

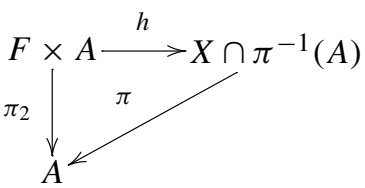

In the diagram above $\pi_{2}: F \times A \rightarrow A$ is the projection onto the second factor. We call $h$ a definable trivialization of $X$ over $A$.

If $Y$ is a definable subset of $X$, we say that the trivialization $h$ is compatible with $Y$ if there is a definable subset $G$ of $F$ such that $h(G \times A)=Y \cap \pi^{-1}(A)$. Clearly, the restriction of $h$ to $G \times A$ is a trivialization of $Y$ over $A$.

Theorem 4.2 (Hardt's theorem for definable families) Let $X \subset \mathbb{R}^{k} \times \mathbb{R}^{\ell}$ be a definable set and let $Y_{1}, \ldots, Y_{m}$ be definable subsets of $X$. Then, there exists a finite partition of $\mathbb{R}^{\ell}$ into definable sets $C_{1}, \ldots, C_{N}$ such that $X$ is definably trivial over each $C_{i}$, and moreover the trivializations over each $C_{i}$ are compatible with $Y_{1}, \ldots, Y_{m}$.

Remark 4.3 We first remark that it is straightforward to derive from the proof of Theorem 4.2 that the definable sets $C_{1}, \ldots, C_{N}$ can be chosen to be locally closed, and can be expressed as $C_{1}=\mathbb{R}^{\ell} \backslash B_{1}, C_{2}=B_{1} \backslash B_{2}, \ldots, C_{N}=B_{N-1} \backslash B_{N}$ for closed definable sets $B_{1}, \ldots, B_{N}$. Clearly, the closed definable sets $B_{1}, \ldots, B_{N}$, determine the sets $C_{i}$ of the partition.

Remark 4.4 Note also that it follows from Theorem 4.2, that there are only a finite number of topological types among the fibers of any definable map $f: X \rightarrow Y$ between definable sets $X$ and $Y$. This remark will be used a number of times later in the paper. 
Since in what follows we will need to consider many different projections, we adopt the following convention.

Notation 4.5 Given $m$ and $p, p \leq m$, we will denote by

$$
\pi_{m}^{\leq p}: \mathbb{R}^{m} \rightarrow \mathbb{R}^{p}
$$

(respectively, $\pi_{m}^{>p}: \mathbb{R}^{m} \rightarrow \mathbb{R}^{m-p}$ ) the projection onto the first $p$ (respectively, the last $m-p$ ) coordinates.

\subsection{Definable Triangulations}

A triangulation of a closed and bounded definable set $S$ is a simplicial complex $\Delta$ together with a definable homeomorphism from $|\Delta|$ to $S$. Given such a triangulation we will often identify the simplices in $\Delta$ with their images in $S$ under the given homeomorphism.

We call a triangulation $h_{1}:\left|\Delta_{1}\right| \rightarrow S$ of a definable set $S$, to be a refinement of a triangulation $h_{2}:\left|\Delta_{2}\right| \rightarrow S$ if for every simplex $\sigma_{1} \in \Delta_{1}$, there exists a simplex $\sigma_{2} \in \Delta_{2}$ such that $h_{1}\left(\left|\sigma_{1}\right|\right) \subset h_{2}\left(\left|\sigma_{2}\right|\right)$.

Let $S_{1} \subset S_{2}$ be two closed and bounded definable subsets of $\mathbb{R}^{k}$. We say that a definable triangulation $h:|\Delta| \rightarrow S_{2}$ of $S_{2}$, respects $S_{1}$ if for every simplex $\sigma \in \Delta$, $h(\sigma) \cap S_{1}=h(\sigma)$ or $\emptyset$. In this case, $h^{-1}\left(S_{1}\right)$ is identified with a subcomplex of $\Delta$ and $\left.h\right|_{h^{-1}\left(S_{1}\right)}: h^{-1}\left(S_{1}\right) \rightarrow S_{1}$ is a definable triangulation of $S_{1}$. We will refer to this subcomplex by $\left.\Delta\right|_{S_{1}}$.

We introduce the following notational conventions in order to simplify arguments used later in the paper.

Notation 4.6 If $T \subset \mathbb{R}^{k_{1}+k_{2}+\ell}$ be any definable subset of $\mathbb{R}^{k_{1}+k_{2}+\ell}$, for each $m \geq 0$, and $\left(\mathbf{z}, \mathbf{y}_{0}, \ldots, \mathbf{y}_{m}\right) \in \mathbb{R}^{k_{2}+(m+1) \ell}$, we will denote by $T_{\mathbf{z}, \mathbf{y}_{0}, \ldots, \mathbf{y}_{m}} \subset \mathbb{R}^{k_{1}}$ the definable set $\bigcup_{0 \leq i \leq m}\left\{\mathbf{x} \in \mathbb{R}^{k_{1}} \mid(\mathbf{x}, \mathbf{z}) \in T_{\mathbf{y}_{i}}\right\}$. For $\left\{j_{0}, \ldots, j_{m^{\prime}}\right\} \subset[m]$, we will denote by $\pi_{m, j_{0}, \ldots, j_{m^{\prime}}}: \mathbb{R}^{(m+1) \ell} \rightarrow \mathbb{R}^{\left(m^{\prime}+1\right) \ell}$ the projection map on the appropriate blocks of coordinates.

It is well known that compact definable sets are triangulable; moreover, the usual proof of this fact (see for instance [6]) can be easily extended to produce a definable triangulation in a parameterized way. We will actually need a family of such triangulations satisfying certain compatibility conditions that were mentioned earlier. The following proposition states the existence of such families. We omit the proof of the proposition since it is a technical but straightforward extension of the proof of existence of triangulations for definable sets.

Proposition 4.7 (Existence of $m$-adaptive triangulations) Let $T \subset \mathbb{R}^{k_{1}+k_{2}+\ell}$ be a closed and bounded definable subset of $\mathbb{R}^{k_{1}+k_{2}+\ell}$ and let $m \geq 0$. For each $0 \leq p \leq m$, there exists:

(1) A definable partition $\left\{C_{p, \alpha}\right\}_{\alpha \in I_{p}}$ of $\mathbb{R}^{k_{2}+(p+1) \ell}$, into locally closed sets, determined by a sequence of definable closed sets, $\left\{B_{p, \alpha}\right\}_{\alpha \in I_{p}}$ (see Remark 4.3). 
(2) For each $\alpha \in I_{p}$, a definable continuous map,

$$
h_{p, \alpha}:\left|\Delta_{p, \alpha}\right| \times C_{p, \alpha} \rightarrow \bigcup_{\left(\mathbf{z}, \mathbf{y}_{0}, \ldots, \mathbf{y}_{p}\right) \in C_{p, \alpha}} T_{\mathbf{z}, \mathbf{y}_{0}, \ldots, \mathbf{y}_{p}},
$$

where $\Delta_{p, \alpha}$ is a simplicial complex, and such that for each $\left(\mathbf{z}, \mathbf{y}_{0}, \ldots, \mathbf{y}_{p}\right) \in$ $C_{p, \alpha}$, the restriction of $h_{p, \alpha}$ to $\left|\Delta_{p, \alpha}\right| \times\left(\mathbf{z}, \mathbf{y}_{0}, \ldots, \mathbf{y}_{p}\right)$ is a definable triangulation

$$
h_{p, \alpha}:\left|\Delta_{p, \alpha}\right| \times\left(\mathbf{z}, \mathbf{y}_{0}, \ldots, \mathbf{y}_{p}\right) \rightarrow T_{\mathbf{z}, \mathbf{y}_{0}, \ldots, \mathbf{y}_{p}}
$$

of the definable set $T_{\mathbf{z}, \mathbf{y}_{0}, \ldots, \mathbf{y}_{p}}$ respecting the subsets, $T_{\mathbf{z}, \mathbf{y}_{0}}, \ldots, T_{\mathbf{z}, \mathbf{y}_{p}}$.

(3) For each subset $\left\{j_{0}, \ldots, j_{p^{\prime}}\right\} \subset[p],\left(\operatorname{Id}_{k_{2}}, \pi_{p, j_{0}, \ldots, j_{p^{\prime}}}\right)\left(C_{p, \alpha}\right) \subset C_{p^{\prime}, \beta}$ for some $\beta \in I_{p^{\prime}}$, and for each $\left(\mathbf{z}, \mathbf{y}_{0}, \ldots, \mathbf{y}_{p}\right) \in C_{p, \alpha}$, the definable triangulation of $T_{\mathbf{z}, \mathbf{y}_{j_{0}}, \ldots, \mathbf{y}_{j_{p^{\prime}}}}$ induced by the triangulation

$$
h_{p, \alpha}:\left|\Delta_{p, \alpha}\right| \times\left(\mathbf{z}, \mathbf{y}_{0}, \ldots, \mathbf{y}_{p}\right) \rightarrow T_{\mathbf{z}, \mathbf{y}_{0}, \ldots, \mathbf{y}_{p}}
$$

is a refinement of the definable triangulation,

$$
h_{p^{\prime}, \beta}:\left|\Delta_{p^{\prime}, \beta}\right| \times\left(\mathbf{z}, \mathbf{y}_{j_{0}}, \ldots, \mathbf{y}_{j_{p^{\prime}}}\right) \rightarrow T_{\mathbf{z}, \mathbf{y}_{j_{0}}, \ldots, \mathbf{y}_{j_{p^{\prime}}}} .
$$

(We will call the family $\left\{h_{p, \alpha}\right\}_{0 \leq p \leq m, \alpha \in I_{p}}$ an $m$-adaptive family of triangulations of $T$.

We will also need the following technical result.

Proposition 4.8 Let $C_{t} \subset \mathbb{R}^{k}, t \geq 0$ be a definable family of closed and bounded sets, and let $C \subset \mathbb{R}^{k+1}$ be the definable set $\bigcup_{t \geq 0} C_{t} \times\{t\}$. If for every $0 \leq t<t^{\prime}$, $C_{t} \subset C_{t^{\prime}}$, and $C_{0}=\pi_{k+1}^{\leq k}\left(\bar{C} \cap \mathbb{R}^{k} \times\{0\}\right)$, then there exists $t_{0}>0$ such that $C_{0}$ has the same homotopy type as $C_{t}$ for every $t$ with $0 \leq t \leq t_{0}$.

Proof The proof given in [5] (see Lemma 16.17) for the semi-algebraic case can be easily adapted to the o-minimal setting using Hardt's triviality for definable families instead of for semi-algebraic ones.

We now introduce another notational convention.

Notation 4.9 Let $\mathcal{F}(x)$ be a predicate defined over $\mathbb{R}_{+}$and $y \in \mathbb{R}_{+}$. The notation $\forall(0<x \ll y) \mathcal{F}(x)$ stands for the statement

$$
\exists z \in(0, y) \quad \forall x \in \mathbb{R}_{+} \quad(\text { if } x<z, \text { then } \mathcal{F}(x)),
$$

and can be read "for all positive $x$ sufficiently smaller than $y, \mathcal{F}(x)$ is true".

More generally, 
Fig. 1 The complex $\Delta_{[n]}$

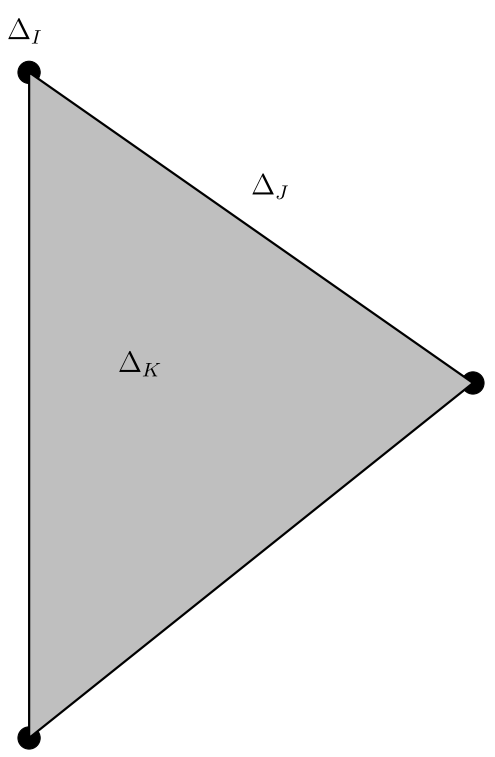

Notation 4.10 For $\bar{\varepsilon}=\left(\varepsilon_{0}, \ldots, \varepsilon_{n}\right)$ and a predicate $\mathcal{F}(\bar{\varepsilon})$ over $\mathbb{R}_{+}^{n}$ we say "for all sufficiently small $\bar{\varepsilon}, \mathcal{F}(\bar{\varepsilon})$ is true" if

$$
\forall\left(0<\varepsilon_{0} \ll 1\right) \forall\left(0<\varepsilon_{1} \ll \varepsilon_{0}\right) \cdots \forall\left(0<\varepsilon_{n} \ll \varepsilon_{n-1}\right) \mathcal{F}(\bar{\varepsilon}) .
$$

\subsection{Infinitesimal Thickenings of the Faces of a Simplex}

We will need the following construction.

Let $\bar{\varepsilon}=\left(\varepsilon_{0}, \ldots, \varepsilon_{n}\right) \in \mathbb{R}_{+}^{n+1}$, with $0 \leq \varepsilon_{n}<\cdots<\varepsilon_{0}<1$. Later we will require $\bar{\varepsilon}$ to be sufficiently small (see Notation 4.10).

For a face $\Delta_{J} \in \Delta_{[n]}$, we denote by $C_{J}(\bar{\varepsilon})$ the subset of $\left|\Delta_{J}\right|$ defined by

$$
C_{J}(\bar{\varepsilon})=\left\{x \in\left|\Delta_{J}\right| \mid \operatorname{dist}\left(x,\left|\Delta_{I}\right|\right) \geq \varepsilon_{\# I-1} \text { for all } I \subset J\right\} .
$$

Note that,

$$
\left|\Delta_{[n]}\right|=\bigcup_{I \subset[n]} C_{I}(\bar{\varepsilon}) .
$$

Also, observe that for sufficiently small $\bar{\varepsilon}>0$, the various $C_{J}(\bar{\varepsilon})$ 's are all homeomorphic to closed balls, and moreover all nonempty intersections between them also have the same property. Thus, the cells $C_{J}(\bar{\varepsilon})$ 's together with the nonempty intersections between them form a regular cell complex, $\mathcal{C}\left(\Delta_{[n]}, \bar{\varepsilon}\right)$, whose underlying topological space is $\left|\Delta_{[n]}\right|$ (see Figs. 1 and 2).

Definition 4.11 We will denote by $\mathcal{C}\left(\operatorname{sk}_{m}\left(\Delta_{[n]}\right), \bar{\varepsilon}\right)$ the subcomplex of $\mathcal{C}\left(\Delta_{[n]}, \bar{\varepsilon}\right)$ consisting of the cells $C_{I}(\bar{\varepsilon})$ 's together with the nonempty intersections between them where $|I| \leq m+1$. 
Fig. 2 The corresponding complex $\mathcal{C}\left(\Delta_{[n]}\right)$ with $I \subset J \subset K=[n]$

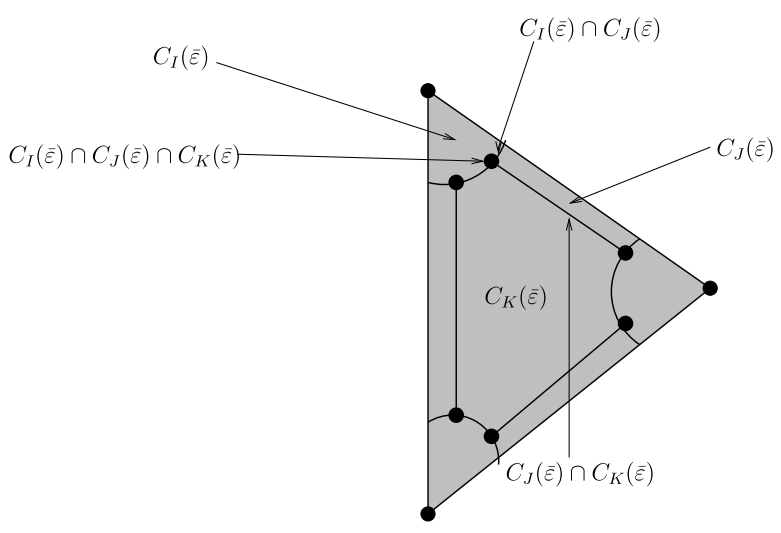

$I \subset J \subset K=[n]$

We now use thickened simplices defined above to define a thickened version of the homotopy co-limit of an arrangement $\mathcal{A}$.

\subsection{Thickened Homotopy Co-limits}

Given an $m$-adaptive family of triangulations of $T$ (cf. Proposition 4.7), $\left\{h_{p, \alpha}\right\}_{0 \leq p \leq m, \alpha \in I_{p}}$ and $\mathbf{z} \in \mathbb{R}^{k_{2}}$, we define a cell complex, $\operatorname{hocolim}_{m}^{+}\left(\mathcal{A}_{\mathbf{z}}, \bar{\varepsilon}\right)$ (best thought of as an infinitesimally thickened version of $\left.\operatorname{hocolim}_{m}\left(\mathcal{A}_{\mathbf{z}}, \bar{\varepsilon}\right)\right)$, whose associated topological space is homotopy equivalent to $\left|\operatorname{hocolim}_{m}\left(\mathcal{A}_{\mathbf{z}}\right)\right|$.

Definition 4.12 (The cell complex hocolim ${ }_{m}^{+}\left(\mathcal{A}_{\mathbf{z}}, \bar{\varepsilon}\right)$ ) Let $\mathcal{C}_{m}$ denote the cell complex $\mathcal{C}\left(\operatorname{sk}_{m}\left(\Delta_{[n]}\right), \bar{\varepsilon}\right)$ defined previously (cf. Definition 4.11).

Let $C$ be a cell of $\mathcal{C}_{m}$. Then, $C \subset\left|\Delta_{I}\right|$ for a unique simplex $\Delta_{I}$ with $I=\left\{i_{0}, \ldots, i_{m^{\prime}}\right\} \subset[n], m^{\prime} \leq m$, and (following notation introduced before in Definition 4.11)

$$
C=C_{I_{1}}(\bar{\varepsilon}) \cap \cdots \cap C_{I_{p}}(\bar{\varepsilon}),
$$

with $I_{1} \subset I_{2} \subset \cdots \subset I_{p} \subset I$ and $p \leq m^{\prime}$.

We denote by $\mathcal{K}(C, \bar{\varepsilon})$ the cell complex consisting of the cells

$$
C \times h_{m^{\prime}, \alpha}\left(|\sigma|, \mathbf{z}, \mathbf{y}_{i_{0}}, \ldots, \mathbf{y}_{i_{m^{\prime}}}\right)
$$

with $\alpha \in I_{m^{\prime}},\left(\mathbf{z}, \mathbf{y}_{i_{0}}, \ldots, \mathbf{y}_{i_{m^{\prime}}}\right) \in C_{\alpha, m^{\prime}}, \sigma \in \Delta_{m^{\prime}, \alpha}$, and $h_{m^{\prime}, \alpha}\left(|\sigma|, \mathbf{z}, \mathbf{y}_{i_{0}}, \ldots, \mathbf{y}_{i_{m^{\prime}}}\right) \subset$ $\mathcal{A}_{\mathbf{z}, I}$. We denote

$$
\operatorname{hocolim}_{m}^{+}\left(\mathcal{A}_{\mathbf{z}}, \bar{\varepsilon}\right)=\bigcup_{C \in \mathcal{C}_{m}} \mathcal{K}(C) .
$$

The compatibility properties (properties (2) and (3) in Proposition 4.7) of the $m$-adaptive family of triangulations of $T,\left\{h_{p, \alpha}\right\}_{0 \leq p \leq m, \alpha \in I_{p}}$, ensure that $\operatorname{hocolim}_{m}^{+}\left(\mathcal{A}_{\mathbf{z}}, \bar{\varepsilon}\right)$ defined above is a regular cell complex. Notice that, since the map 
$f_{\mathcal{A}}$ defined in (3.1) extends to $\left|\operatorname{hocolim}_{m}^{+}\left(\mathcal{A}_{\mathbf{z}}, \bar{\varepsilon}\right)\right|$, the notion of diagram-preserving maps extend to $\mid$ hocolim $_{m}^{+}\left(\mathcal{A}_{\mathbf{z}}, \bar{\varepsilon}\right) \mid$ as well.

We now prove:

Lemma 4.13 Let $\mathbf{z} \in \mathbb{R}^{\ell}$ and $m \geq 0$. Then, for all sufficiently small $\bar{\varepsilon}>0$, $\left|\operatorname{hocolim}_{m}^{+}\left(\mathcal{A}_{\mathbf{z}}, \bar{\varepsilon}\right)\right|$ is homotopy equivalent to $\left|\operatorname{hocolim}_{m}\left(\mathcal{A}_{\mathbf{z}}\right)\right|$ by a diagram-preserving homotopy equivalence.

Proof Let $N=\mid$ hocolim ${ }_{m}^{+}\left(\mathcal{A}_{\mathbf{z}}, \bar{\varepsilon}\right) \mid$. First replace $\varepsilon_{m}$ by a variable $t$ in the definition of $N$ to obtain a closed and bounded definable set, $N_{t}^{m}$, and observe that $N_{t}^{m} \subset N_{t^{\prime}}^{m}$ for all $0<t<t^{\prime} \ll 1$.

Now apply Proposition 4.8 to obtain that $N$ is homotopy equivalent to $N_{0}^{m}$. Now, replace $\varepsilon_{m-1}$ by $t$ in the definition of $N_{0}^{m}$ to obtain $N_{t}^{m-1}$, and applying Proposition 4.8 obtain that $N_{0}^{m}$ is homotopy equivalent to $N_{0}^{m-1}$. Continuing in this way we finally obtain that, $N$ is homotopy equivalent to $N_{0}^{0}=\left|\operatorname{hocolim}_{m}\left(\mathcal{A}_{z}\right)\right|$. Moreover, the diagram-preserving property is clearly preserved at each step of the proof.

Proof of Theorem 1.8 Recall that for $m \geq 0$, and $\left(\mathbf{z}, \mathbf{y}_{0}, \ldots, \mathbf{y}_{m}\right) \in \mathbb{R}^{k_{2}+(m+1) \ell}$, we denote by $T_{\mathbf{z}, \mathbf{y}_{0}, \ldots, \mathbf{y}_{m}}$ the definable set

$$
\bigcup_{i=1}^{m} T_{\mathbf{z}, \mathbf{y}_{i}} \subset \mathbb{R}^{\ell}
$$

Now apply Proposition 4.7 to the set $T$ with $m=k_{1}$ to obtain an $k_{1}$-adaptive family of triangulations $\left\{h_{p, \alpha}\right\}_{1 \leq p \leq k_{1}, \alpha \in I_{p}}$.

We now fix $\left\{\mathbf{y}_{1}, \ldots, \mathbf{y}_{n}\right\} \subset \mathbb{R}^{\ell}$ and let $\mathcal{A}=\left\{A_{1}, \ldots, A_{n}\right\}$ with $A_{i}=T_{\mathbf{y}_{i}} \subset \mathbb{R}^{k_{1}+k_{2}}$. For each $\mathbf{z} \in \mathbb{R}^{k_{2}}$, we will denote by $\mathcal{A}_{z}=\left\{A_{1, \mathbf{z}}, \ldots, A_{n, \mathbf{z}}\right\}$ where $A_{i, \mathbf{z}}=\{\mathbf{x} \in$ $\left.\mathbb{R}^{k_{1}} \mid(\mathbf{x}, \mathbf{z}) \in A_{i}\right\}$.

For $\alpha \in I_{k_{1}}$, and $1 \leq i_{0}<\cdots<i_{k_{1}} \leq n$, we will denote by $B_{k_{1}, \alpha, i_{0}, \ldots, i_{k_{1}}} \subset \mathbb{R}^{k_{2}}$ the definable closed set

$$
B_{k_{1}, \alpha, i_{0}, \ldots, i_{k_{1}}}=\left\{\mathbf{z} \in \mathbb{R}^{k_{2}} \mid\left(\mathbf{z}, \mathbf{y}_{0}, \ldots, \mathbf{y}_{k_{1}}\right) \in B_{k_{1}, \alpha}\right\} .
$$

Let

$$
\mathcal{B}=\bigcup_{\alpha \in I_{k_{1}}}\left\{B_{k_{1}, \alpha, i_{0}, \ldots, i_{k_{1}}} \mid 1 \leq i_{0}<i_{1}<\cdots<i_{k_{1}} \leq n\right\},
$$

and let $C \in \mathcal{C}(\mathcal{B})$. Theorem 1.8 will follow from the following two lemmas.

Lemma 4.14 For any $\mathbf{z}_{1}, \mathbf{z}_{2} \in C, \mathcal{A}_{\mathbf{z}_{1}}$ is stable homotopy equivalent to $\mathcal{A}_{\mathbf{z}_{2}}$.

Proof Clearly, by Theorem 3.6 it suffices to prove that $\left|\operatorname{hocolim}_{k_{1}}\left(\mathcal{A}_{\mathbf{z}_{1}}\right)\right|$ is diagrampreserving homotopy equivalent to $\left|\operatorname{hocolim}_{k_{1}}\left(\mathcal{A}_{\mathbf{z}_{2}}\right)\right|$.

The compatibility properties of the triangulations ensure that the complex $\left|\operatorname{hocolim}_{k_{1}}^{+}\left(\mathcal{A}_{\mathbf{z}_{1}}, \bar{\varepsilon}\right)\right|$ is isomorphic to $\left|\operatorname{hocolim}_{k_{1}}^{+}\left(\mathcal{A}_{\mathbf{z}_{2}}, \bar{\varepsilon}\right)\right|$ and hence $\left|\operatorname{hocolim}_{k_{1}}^{+}\left(\mathcal{A}_{\mathbf{z}_{1}}, \bar{\varepsilon}\right)\right|$ is homeomorphic to $\left|\operatorname{hocolim}_{k_{1}}^{+}\left(\mathcal{A}_{\mathbf{z}_{1}}, \bar{\varepsilon}\right)\right|$. 
Using Lemma 4.13 we get a diagram-preserving homotopy equivalence

$$
\phi:\left|\operatorname{hocolim}_{k_{1}}\left(\mathcal{A}_{\mathbf{z}_{1}}\right)\right| \rightarrow\left|\operatorname{hocolim}_{k_{1}}\left(\mathcal{A}_{\mathbf{z}_{2}}\right)\right| \text {. }
$$

It now follows from Theorem 3.6 that the arrangements $\mathcal{A}_{\mathbf{z}_{1}}$ and $\mathcal{A}_{\mathbf{z}_{2}}$ are stable homotopy equivalent.

Lemma 4.15 There exists a constant $C(T)$ such that the cardinality of $\mathcal{C}(\mathcal{B})$ is bounded by $C \cdot n^{\left(k_{1}+1\right) k_{2}}$.

Proof Notice that each $B_{k_{1}, \alpha}, \alpha \in I_{k_{1}}$ is a definable subset of $\mathbb{R}^{k_{2}+\left(k_{1}+1\right) \ell}$ depending only on $T$. Also, the cardinality of the index set $I_{k_{1}}$ is determined by $T$.

Hence the set $\mathcal{B}$ consists of $C(T) \cdot\left(\begin{array}{c}n \\ k_{1}+1\end{array}\right)$ definable sets each one of them is a

$$
\left(B_{k_{1}, \alpha}, \pi_{k_{2}+\left(k_{1}+1\right) \ell}^{\leq k_{2}}, \pi_{k_{2}+\left(k_{1}+1\right) \ell}^{>k_{2}}\right)
$$

for some $\alpha \in I_{k_{1}}$. Here $C(T)>0$ is a constant that depends only on $T$. Using Observation 4.1 , we have that $\mathcal{B}$ is a $\left(B, \pi_{1}^{\prime}, \pi_{2}^{\prime}\right)$-set for some $B$ determined only by $T$. Now apply Theorem 2.2.

The theorem now follows from Lemmas 4.14 and 4.15 proved earlier.

Proof of Theorem 1.9 The proof is similar to that of Theorem 1.8 given above, except we use Theorem 3.7 instead of Theorem 3.6, and this accounts for the slight worsening of the exponent in the bound.

Proof of Theorem 2.7 Using a construction due to Gabrielov and Vorobjov [9] (see also [2]) it is possible to replace any given $\mathcal{A}$-set by a closed bounded $\mathcal{A}^{\prime}$-set (where $\mathcal{A}^{\prime}$ is a new family of definable closely related to $\mathcal{A}$ with $\# \mathcal{A}^{\prime}=2 k(\# \mathcal{A})$ ), such that the new set has the same homotopy type as the original one. Using this construction one can directly deduce Theorem 2.7 from Theorem 1.9 . We omit the details.

\section{References}

1. Agarwal, P.K., Sharir, M.: In: Urrutia, J., Sack, J.R. (eds.) Arrangements and Their Applications. Handbook of Computational Geometry, pp. 49-119. North-Holland, Amsterdam (2000). MR 1746675

2. Basu, S.: Combinatorial complexity in o-minimal geometry. Preprint at arXiv:math.CO/0612050 (2006). An extended abstract appears in the Proceedings of the ACM Symposium on the Theory of Computing (2007)

3. Basu, S.: Algorithmic Semi-algebraic Geometry and Topology-Recent Progress and Open Problems. Surveys on Discrete and Computational Geometry: Twenty Years Later, Contemporary Mathematics, vol. 453, pp. 139-212. American Mathematical Society, Providence (2008)

4. Basu, S., Vorobjov, N.: On the number of homotopy types of fibres of a definable map. J. Lond. Math. Soc. (2) 76(3), 757-776 (2007). MR 2377123

5. Basu, S., Pollack, R., Roy, M.-F.: Algorithms in Real Algebraic Geometry, Algorithms and Computation in Mathematics, 2nd edn., vol. 10. Springer, Berlin (2006). MR 1998147 (2004g:14064)

6. Coste, M.: An introduction to o-minimal geometry. Istituti Editoriali e Poligrafici Internazionali, Pisa, Dip. Mat. Univ. Pisa, Dottorato di Ricerca in Matematica (2000) 
7. Danzer, L., Grünbaum, B., Klee, V.: Helly's Theorem and Its Relatives. Proc. Sympos. Pure Math., vol. VII, pp. 101-180. Am. Math. Soc., Providence (1963). MR 0157289 (28 \#524)

8. Dieudonné, J.: A History of Algebraic and Differential Topology. 1900-1960. Birkhäuser Boston, Boston (1989). MR 995842 (90g:01029)

9. Gabrielov, A., Vorobjov, N.: Approximation of definable sets by compact families and upper bounds on homotopy and homology. Preprint at arXiv:math.AG/0710.3028v1 (2007)

10. Grigor'ev, D.Yu., Vorobjov, N.N. Jr.: Solving systems of polynomial inequalities in subexponential time. J. Symb. Comput. 5(1-2), 37-64 (1988). MR 949112 (89h:13001)

11. Knight, J.F., Pillay, A., Steinhorn, C.: Definable sets in ordered structures. II. Trans. Am. Math. Soc. 295(2), 593-605 (1986). MR 0833698 (88b:03050b)

12. Matoušek, J.: Lectures on Discrete Geometry. Graduate Texts in Mathematics, vol. 212, Springer, New York (2002). MR 1899299 (2003f:52011)

13. Pillay, A., Steinhorn, C.: Definable sets in ordered structures. I. Trans. Am. Math. Soc. 295(2), 565592 (1986). MR 0833697 (88b:03050a)

14. Pillay, A., Steinhorn, C.: Definable sets in ordered structures. III. Trans. Am. Math. Soc. 309(2), 469-576 (1988). MR 0943306 (89i:03059)

15. Rolin, J.-P., Speissegger, P., Wilkie, A.J.: Quasianalytic Denjoy-Carleman classes and o-minimality. J. Am. Math. Soc. 16(4), 751-777 (2003), (electronic). MR 1992825 (2004g:14065)

16. Smale, S.: A Vietoris mapping theorem for homotopy. Proc. Am. Math. Soc. 8, 604-610 (1957). MR $0087106(19,302 \mathrm{f})$

17. Spanier, E.H.: Algebraic Topology. McGraw-Hill, New York (1966). MR 0210112 (35 \#1007)

18. Spanier, E.H., Whitehead, J.H.C.: Duality in relative homotopy theory. Ann. Math. (2) 67, 203-238 (1958). MR 0105105 (21 \#3850)

19. van den Dries, L.: Remarks on Tarski's Problem Concerning $(\mathbf{R},+, \cdot$, exp), Logic Colloquium '82, Florence, 1982. Stud. Logic Found. Math., vol. 112, pp. 97-121. North-Holland, Amsterdam (1984). MR 762106 (86g:03052)

20. van den Dries, L.: Tame Topology and O-Minimal Structures. London Mathematical Society Lecture Note Series, vol. 248. Cambridge University Press, Cambridge (1998). MR 1633348 (99j:03001)

21. van den Dries, L., Miller, C.: Geometric categories and o-minimal structures. Duke Math. J. 84(2), 497-540 (1996). MR 1404337 (97i:32008)

22. van den Dries, L., Speissegger, P.: The real field with convergent generalized power series. Trans. Am. Math. Soc. 350(11), 4377-4421 (1998). MR 1458313 (99a:03036)

23. van den Dries, L., Speissegger, P.: The field of reals with multisummable series and the exponential function. Proc. Lond. Math. Soc. (3) 81(3), 513-565 (2000). MR 1781147 (2002k:03057)

24. Viro, O.Ya., Fuchs, D.B.: Homology and Cohomology. Topology. II, Encyclopaedia Math. Sci., vol. 24. Springer, Berlin (2004). Translated from the Russian by C.J. Shaddock, pp. 95-196. MR 2054457

25. Whitehead, G.W.: Elements of Homotopy Theory. Graduate Texts in Mathematics, vol. 61. Springer, New York (1978). MR 516508 (80b:55001)

26. Wilkie, A.J.: Model completeness results for expansions of the ordered field of real numbers by restricted Pfaffian functions and the exponential function. J. Am. Math. Soc. 9(4), 1051-1094 (1996). MR 1398816 (98j:03052)

27. Wilkie, A.J.: A theorem of the complement and some new o-minimal structures. Selecta Math. (N.S.) 5(4), 397-421 (1999). MR 1740677 (2001c:03071) 